\title{
Application of short time energy analysis in monitoring the stability of arc sound signal
}

\author{
Ping Yao ${ }^{\mathrm{a}, \mathrm{b}}$, Kang Zhou ${ }^{\mathrm{c}, *}$ \\ a School of Electrical and Mechanical, Guangdong Polytechnic Normal University, China \\ ${ }^{\mathrm{b}}$ School of Mechanical and Automotive Engineering, South China University of Technology, China \\ ${ }^{\mathrm{c}}$ State Key Laboratory of High-temperature Gas Dynamics, Institute of Mechanics, Chinese Academy of Sciences, China
}

\section{A R T I C L E I N F O}

\section{Article history:}

Received 7 June 2016

Received in revised form 7 March 2017

Accepted 10 April 2017

Available online 12 April 2017

\section{Keywords:}

Short time energy

Arc sound signal

Quantitative evaluation

Statistical analysis

\begin{abstract}
A B S T R A C T
This paper employed the short time energy of arc sound signals to online quantitatively describe the stability of arc sound signal. At first, the signal can be preprocessed by wavelet packet filtering, and then detailed information of the short time energy of the signal was obtained using hamming window. After statistical analyzed the short time energy, the energy distribution possibility and cumulative distribution function of the signal can be collected. Then a proposed stability evaluation criterion was employed to quantitatively describe the stability of arc sound signal. Relative experimental data showed that more stable signal corresponded lager value of the criterion. The proposed method which combined the short time energy and statistical analysis was supported by many actual experiments. This contribution can benefit the quantitative evaluation of the arc welding process, and instructed the future parameters optimization to obtain welding products with high quality.
\end{abstract}

(c) 2017 Elsevier Ltd. All rights reserved.

\section{Introduction}

Arc welding is one of the most fundamental process in manufacturing and employed in fabricating metal products in many areas. The welding process monitoring and quality evaluation are important to improve the welding quality and production efficiency [1]. It is considered to be a highly skilled profession and requires long time's experiences. Currently, robotic welding has been successfully employed in industries for many years and given risen to a lot of benefits for global market. The high automation of robotic welding is necessary to improve the efficiency of actual production, such as using computer vision technology to identify and locate the position of welding seams [2], or using pattern recognition to measure the energy consumption of the welding robotics [3], and so on. To achieve the goal, the welding process should be effectively monitored and the stability can be estimated by means of some key process variables.

During arc welding process, arc sound signal is a very important process variable which contains a lot of useful information about welding process [4], and studied by many previous contributions. Lv et al. [5] discovered the relation between arc sound signal and the arc height, through extracting the features from the signals

\footnotetext{
* Corresponding author.

E-mail address: zhoukang326@126.com (K. Zhou).
}

after processed by wavelet analysis and wavelet packet analysis in time domain, and then employed the arc sound signal and artificial neutral network (ANN) to predict the arc height. Also, their other work [6] analyzed the generation and mechanism of sound channel during the arc welding process, and established an equivalent model based on cepstral coefficient through $\mathrm{Z}$ transform, and then concluded that the sound channel can be employed to identify the dynamic welding process. Pal et al. [7] thought the arc sound signal was found to be strongly related to the process parameters and weld quality as that of voltage and current signals, through analyzing the signals in time and frequency domains to correlate them with the various process parameters and metal transfer modes, and then they used it to detect the weld defects. Grad et al. [8] though the arc sound signals contained information about the behavior of the arc column, the molten pool and droplet transfer, and the signal can easily be employed to assess the stability of arc welding process and detect welding conditions resulting in weld defects. Hence, the arc sound signal can have extensive applications in monitoring the stability of the welding process and weld defects. However, though their previous works employed arc sounds to service the application of arc welding, the effective quantitative method was not developed, in other words, the works cannot sufficiently apply the arc sound signal so far. In addition, the works can only employ the arc sound signal as a simply indictor or sensor, meanwhile, the applications required more auxiliary 
support information and tools, and may have some limitations in practical applications.

To more sufficiently apply arc sound signal, convenient mathematical tools should be employed. In reality, arc sound signal is a sound wave with significant characteristics of vibration. Hence, classic tools which were employed to process speeches and other vibration signals can be considered. Recently, short time energy analysis was employed to analyze the high-pitched speech signals, and presented better performance than conventional linear prediction analysis [9]. Also, it can be used to recovery of missing speech packet [10], and discriminate voiced and unvoiced segments of speech signals [11]. Hence, it can be considered as an effective tool to analyze the acoustic signal in practice.

In this paper, we will employ a tool of short time energy analysis to process the collected arc sound signals, due to the signals are typical and include a lot of useful information during the arc welding process. By means of short time energy analysis, the stability of arc sound signal can be effectively extracted using our self-defined estimation criterion. In Section 2, the principle of short time energy of arc sound signal and statistical analysis were introduced. Section 3 introduced the Cumulative Distribution Function (CDF) Statistical Analysis Evaluation, which is a new method for evaluating the stability of the arc sound signal. In Section 4, the integrated experimental setup and arc sound signals preprocessing were shown. After preprocessing the collect original arc sound signals, corresponding experiments were conducted to validate the proposed method. At last, the conclusion and future work were included in Section 5.

\section{Principle of short time energy}

Short time energy analysis is a general method of analyzing acoustic signal in time domain and commonly employed in monitoring and identifying the acoustic signals [11], such as analyzing the animal population through studying their calls [12], or recognizing the music instrument through analyzing chord music [13], and so on.

Because arc sound signals were unstable, relative technologies which were used to process the stable signals cannot be employed. However, according to its individual characteristics, during a short time range of $10-30 \mathrm{~ms}$, this unstable signal can be considered as a stable one. In other word, the signal had characteristics of a stable signal in short time.

The short time energy analysis can be clearly illustrated [13,14] as follows. Firstly, defining the short time energy of one acoustic signal at time $n$ as:

$E_{n}=\sum_{m=-\infty}^{+\infty}[x(m) \omega(n-m)]^{2}=\sum_{m=n-(N-1)}^{n}[x(m) \omega(n-m)]^{2}$,

where $E_{n}$ is the short time energy, $n$ and $m$ are indexes of the frames, $x(m)$ is the arc sound signal after window function is used, $\omega(n)$ is the window function, $N$ is the length of the window function. The window function is a function which actually is a truncation function. According to Eq. (1), the short time energy is a weighted quadratic sum of energies of each frame. When the window function is a rectangular window, the short time energy can be described as:

$E_{n}=\sum_{m=n-(N-1)}^{n} x^{2}(m)$

Under this circumstance, a filter $h(n)$ was introduced, and $h(n)$ $=\omega^{2}(m)$, then:
$E_{n}=\sum_{m=-\infty}^{+\infty} x^{2}(m) h(n-m)=x^{2}(n) h(n)$.

It can be understood that, firstly, obtained the square value of energy of each sample point, then the values could go through a filter, which composed of an impulse response $h(n)$.The output is a time sequence which composed of every short time energy. Fig. 1 showed the principle of the short time energy.

The chosen of $h(n)$ or other window functions determined the calculation of short time energy. In reality, the chosen of $N$, was closely relative to the cycle of acoustic signal.

In general, the larger of $N$, the smoother of the obtained signal, which makes the overall short time energy having few changes, hence, the energy variation cannot be clearly shown. On the other hand, less value of $N$ may induce the lower effect of smoothing. Though the detailed variation of the signal can be detected, the envelope was difficult to clearly present. Hence, the chosen of $N$ was very important. In this work, the hamming window was selected to analyze, whose mathematical description is as follows:

$\omega(n)=0.54-0.46 \cos \left(2 \pi \frac{n}{N-1}\right), \quad 0 \leqslant n \leqslant N-1$,

after several trails and corresponding analysis, the value of $N$ was chosen as 128 due to this length can obtain more satisfactory results.

Then the short time energy analysis results of signal in Fig. 2(a), which was an original collected arc sound signal, can be shown in Fig. 2(b). It can be seen that the energy began to increase from $0.45 \mathrm{~s}$ to $0.92 \mathrm{~s}$, and then began to decline.

According to Fig. 2, it can be seen that the figure of the short time energy presented the signal varying information in real time. The short time energy variation characteristic of the signal can be clearly shown. However, it cannot show the varying information during the whole process, as well as it is difficult to quantitatively evaluate the variation.

To clearly present the varying information during a whole arc welding process, statistical knowledge can be considered. In this work, the possibility distribution of the short time energy can be calculated as follows:

$P(a \leqslant x<b)=\int_{a}^{b} f(x) d x$

where $a$ and $b$ are respectively the lower boundary and upper boundary of short time energy, $f(x)$ is the short time energy function. In general, the distribution of the short time energy can be clearly presented by histogram of possibility of short time energy. According to relative analysis [10], more stable signal corresponds higher possibility of small values of the short time energy appearing; if the signal is unstable, large value of the short time energy is easy to appear, which means the distribution of the possibilities is more unfocused.

Though the possibility can show the distribution information of the short time energy, it cannot intuitively provide the overall distribution situation of the signal under a certain range. Overall distribution situation is generally described by Cumulative distribution function (CDF) of the selected signal.

$\mathrm{CDF}$ is employed to obtain the value of the sum of selected probabilities, which count the number of the values of probabilities

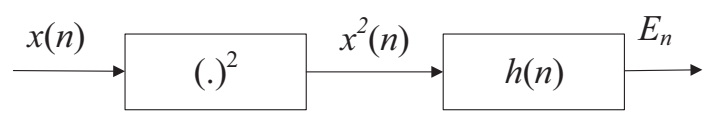

Fig. 1. Principle of short time energy. 

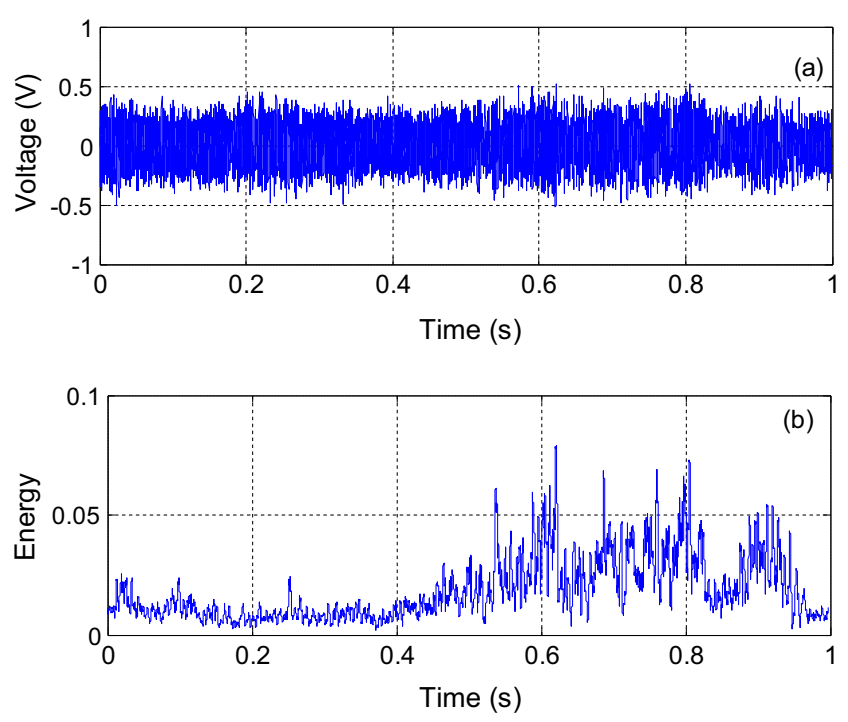

Fig. 2. (a) Original arc sound signal; (b) Short time energy of the signal.

less or equal to a given value, whose mathematical description is shown in Eq. (6) .

$F(x)=P(X \leqslant x)$,

where $F$ is the cumulative distribution value of the short time energy, $x$ is a preliminary given value for special analysis.

CDF was used extensively in system safety assessment [15], image compression [16], wireless data-access scheme [17], and some other relative areas. According to relative analysis [18,19], for a given stable signal, because the majority of energies are low, the corresponding distribution range is small, the ascending trend of the CDF curve may be much steeper, while for a given unstable signal, because the energy distribution range is large, the corresponding probability distribution is much gentler, which induces the ascending trend of the CDF curve may be much smoother. Hence, the CDF curve can be employed to analyze the stability of short time energy of arc sound signal.

In this paper, we proposed a new quantitatively evaluation method, which combined the short time energy and statistics index, for the stability of arc welding process based on arc sound signal analysis.

\section{CDF statistical analysis evaluation based on short time energy}

Above analysis shows short time energy can describe the stability of the arc sound signal. The CDF of the signal can obtain the intuitive overall distribution information under a certain range. In this work, a CDF statistical analysis method based on short time energy was proposed to evaluate the arc sound signal, the detailed procedures were as follows:

Step1: collected the arc sound signals during the welding process;

Step2: preprocessed the collected original signals, separated the noise from the signals through wavelet packet filtering algorithm, and then obtained the arc sound signals with more significant characteristics;

Step3: calculated the short time energy $E_{n}$ of arc sound signals through introducing window function based on Eq. (7):

$E_{n}=\sum_{k=-\infty}^{\infty} x^{2}(k) h(n-k)$;
Step4: calculated the possibility distribution of short time energy, and then plotted the corresponding histogram;

Step5: calculated the cumulative distribution of short time energy, and then plotted the corresponding curve based on Eq. (6). The cumulative distribution should comply with the following rule:

$$
\left\{\begin{array}{l}
\lim _{x \rightarrow-\infty} F(x)=0 \\
\lim _{x \rightarrow+\infty} F(x)=1 \\
F\left(x_{1}\right) \leqslant F\left(x_{2}\right) \quad x_{1} \leqslant x_{2}
\end{array} .\right.
$$

Step6: set proper boundary conditions, obtained the values of slope of CDF curve mentioned in Step5 based on principle of calculus:

$E_{n C D F}=\frac{F\left(x_{1}\right)-F\left(x_{2}\right)}{x_{1}-x_{2}}$,

where $E_{n C D F}$ denotes the slope of CDF curve, $F\left(x_{1}\right)$ and $F\left(x_{2}\right)$ respectively denote the values of the beginning point and ending point of the CDF curve, $x_{1}$ and $x_{2}$ are the corresponding values in the $\mathrm{x}$-axis. In general, if the welding process is stable, the CDF curve may quickly achieve saturation, it means that the sharper slope of the curve indicates much stable of the welding process. Because the beginning and terminate of the curve are not straight lines, to obtain an equivalent linear calculation result, the slope should be calculated excluding the beginning and terminate of the curve. According to a great many relative experiments, it can be observed that when $x_{1}$ and $x_{2}$ respectively correspond the values when Eq. (6) equal to $20 \%$ and $80 \%$ can obtain satisfactory results. Hence, in this work, $x_{1}$ and $x_{2}$ used the values which corresponding $20 \%$ and $80 \%$ in the corresponding $x$-axis.

Step7: obtained the histogram of the possibilities distribution in Step5, and then calculated the value of the possibility of the energy which corresponds the maximum energy distribution possibility under stable condition,

$E_{n P}=P[w(I)]$

where $E_{n P}$ is the maximum possibility in the energy possibility, $w(I)$ is the energy corresponding the maximum energy distribution possibility under stable condition, $I$ is the current used in the welding process. For each array of welding currents, which included the leading current and trailing welding, the value of $w(I)$ could be obtained using sample entropy method for the mathematical fitting results for several experimental results $[20,21]$.

Step8: calculated the self-designed criterion for evaluating the stability of arc sound signal during the arc welding process: $A S S T=E_{n C D F} \times E_{n P}$,

where ASST is the criterion. The two parameters, which were $E_{n C D F}$ and $E_{n P}$, could be used to qualitative the variation tendency of short time energy by means of possibility cumulative distribution and possibility distribution, respectively. For a stable welding process, the values of the two parameters have higher values, which makes the ASST has a higher value. On the other hand, for an unstable welding process, the two parameters restraint mutually, and make the ASST decrease. Hence, it can increase the reliability of the qualitative calculation. This criterion can eliminate the effect of operation process on the stability monitoring and obtain a convinced result. Larger value of ASST denotes more stable welding process.

\section{Experiments and validation}

\subsection{Experiment setup and arc sound signals preprocessing}

To validate the above proposed method, actual arc welding experiments were conducted. The arc sound signals were acquired by a synchronous multi-information acquisition device, which was 
developed by ourselves. In this device, a self-developed welding inverter was employed for a double wire pulsed MIG welding machine. Q235 steel with thickness of $8.0 \mathrm{~mm}$ was used as bead on plate welds. The welding wire employed the H08Mn2Si, and the diameter was $1.0 \mathrm{~mm}$. Pure argon was used as shielding gas with $15 \mathrm{~L} / \mathrm{min}$ of flow rate. The integrated experimental devices can be shown in Fig. 3.

Also, the chemical composition and mechanical properties of the Q235 steel and the H08Mn2Si used in this experiment can be shown in Table 1 [22] and Table 2 [23].

Original arc sound signal was shown in Fig. 2(a), the timescale was $1 \mathrm{~s}$. It can be found that the signal included various noises, so it was difficult to extract the pure characteristic signal from the provided mixed signal. In the work, wavelet packet threshold filtering was employed to preprocess the collected original signal. Wavelet packet decomposition (WPD) is extended from the wavelet decomposition (WD), which is widely used in the area of signal processing [24]. The threshold can be adjusted to an appropriate value to make the noises as few as possible for further processing, according to the principle and characteristics of arc sound signal.

At first, wavelet packet filtering can be employed to preprocess the signal. The preprocessed signal by wavelet packet threshold filtering can be found in Fig. 4, the corresponding original signal was shown in Fig. 2(a). The majority of noises were eliminated after the preprocessing, and the signal were significantly clearer than before. The amplitude of the signal shown in Fig. 4 was obviously lower than that in Fig. 2(a) during the range between 0 and $0.45 \mathrm{~s}$. From $0.45 \mathrm{~s}$ to $0.92 \mathrm{~s}$, the amplitude of the signal increased, which meant that there were interferes or unsteady arc sound sig- nals existing. Through listening to the noise-reduced arc sounds, the sounds were much clearer, at the same time with flip-flop sounds, which denoted splash appeared. It showed that the signal features were well retained after wavelet packet threshold filtering and this technology can be employed to make noise reduce.

Also, we employed the Fast Fourier Transform (FFT) to process the signal, and then obtained the power spectrum density (PSD) of the signal shown in Fig. 4, which can be represented on a logarithmic scale shown in Fig. 5. According to the figure, the arc sound frequencies were generally less than $4000 \mathrm{~Hz}$, and the most power concentrated between 0 and $2000 \mathrm{~Hz}$. Also, the power per hertz above $2000 \mathrm{~Hz}$ was significantly lowered. Hence, in this work, only the signals whose frequencies less than $2000 \mathrm{~Hz}$ were analyzed.

According to above results, the preprocessing of the signal was able to obtain the basic information of the collected signal. Then the newly designed evaluation method mentioned in Section 3 can be introduced to evaluate the overall stability of the arc sound signal.

\subsection{Experiments for signal evaluation}

In the experiments, four signals with different stability levels were employed to analyze. There four original signals, signals after wavelet packet filtering and short time energy were shown in Figs. 6-9, while Fig. 10(a)-(d) were their corresponding possibility distributions and CDF curves.

In Fig. 6 which showed the signal 1, it can be seen that this signal was much stable, the signals waves before and after filtering were uniform. In addition, the short time energy shown in Fig. 6

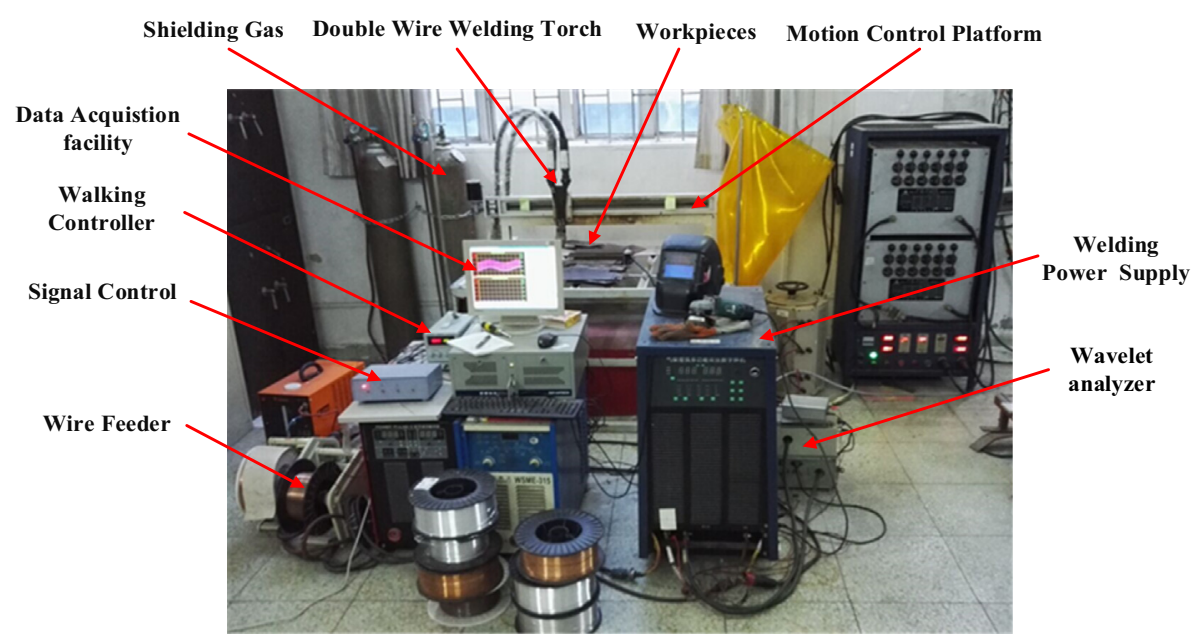

(a)

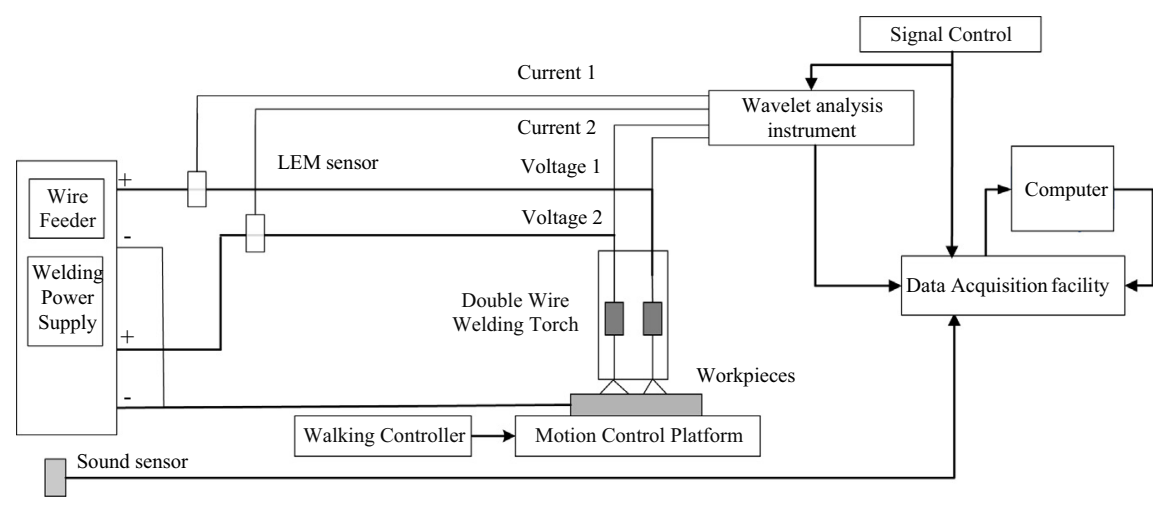

(b)

Fig. 3. Integrated experimental devices used in this work, (a). Actual experimental device, (b) Signal flowchart. 
Table 1

Chemical composition (\%) and mechanical property of Q235 steel.

\begin{tabular}{|c|c|c|c|c|c|c|c|c|}
\hline \multicolumn{5}{|c|}{ Chemical composition $(\%)(\leq)$} & \multicolumn{4}{|c|}{ Mechanical property } \\
\hline $\mathrm{C}$ & Mn & $\mathrm{Si}$ & $\mathrm{S}$ & $\mathrm{P}$ & Density $\left(\mathrm{g} / \mathrm{cm}^{3}\right)$ & Elasticity modulus (E/GPa) & Poisson's ratio (v) & Tensile strength $(\sigma \mathrm{b} / \mathrm{MPa})$ \\
\hline 0.22 & 1.4 & 0.35 & 0.05 & 0.014 & 7.85 & $200-210$ & $0.25-0.33$ & $375-460$ \\
\hline
\end{tabular}

Table 2

Chemical composition $(\%)(\leq)$ of H08Mn2Si.

\begin{tabular}{|c|c|c|c|c|c|c|c|}
\hline $\mathrm{C}$ & $\mathrm{Mn}$ & $\mathrm{Si}$ & $S$ & $\mathrm{P}$ & $\mathrm{Cr}$ & $\mathrm{Ni}$ & $\mathrm{Cu}$ \\
\hline 0.11 & $1.70 / 2.10$ & $0.65 / 0.95$ & 0.035 & 0.035 & 0.20 & 0.30 & 0.20 \\
\hline
\end{tabular}

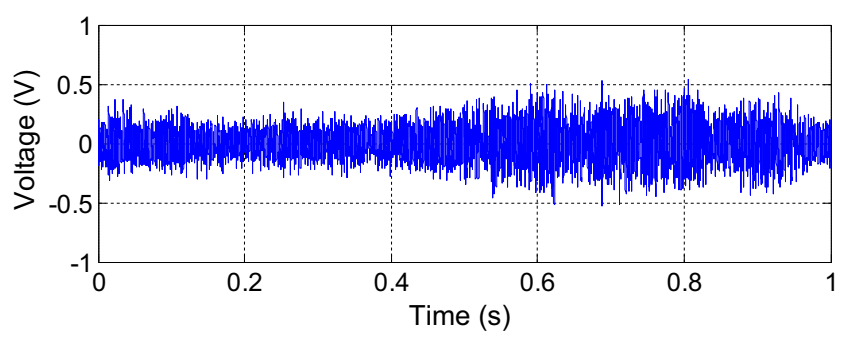

Fig. 4. Preprocessed signal by wavelet packet threshold filtering.

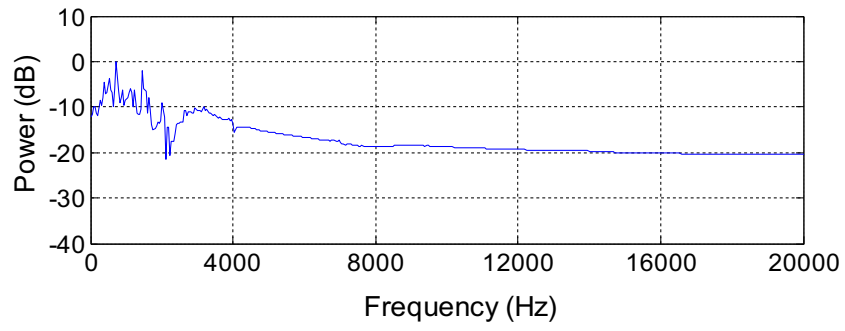

Fig. 5. Power spectrum analysis of the signal.
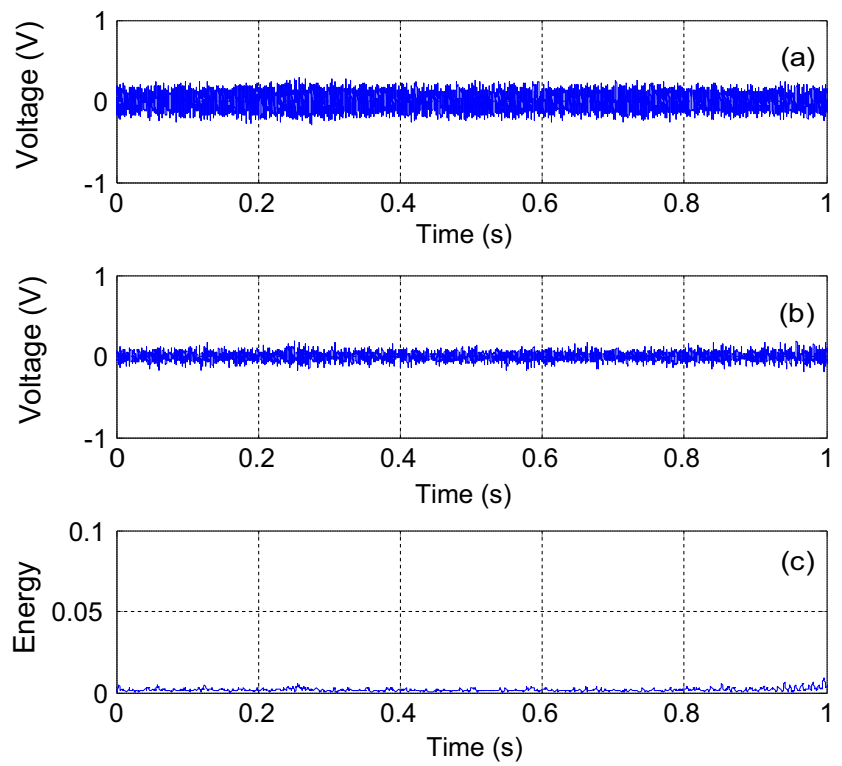

Fig. 6. (a) Original wave of signal 1 , (b) Signal 1 after wavelet packet filtering, (c) Short time energy of signal 1 .
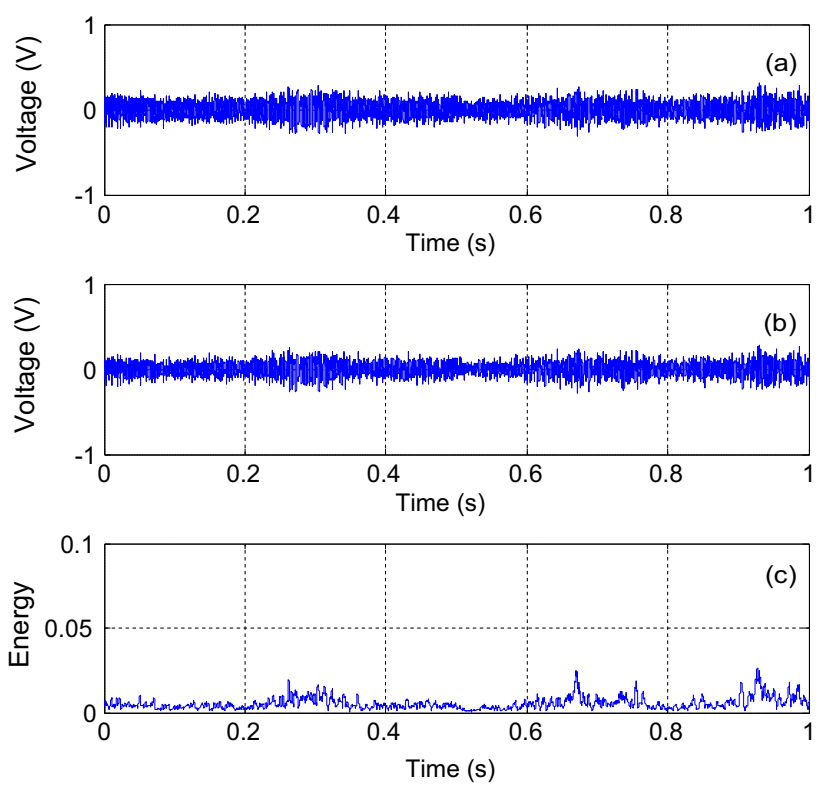

Fig. 7. (a) Original wave of signal 2, (b) Signal 2 after wavelet packet filtering, (c) Short time energy of signal 2.
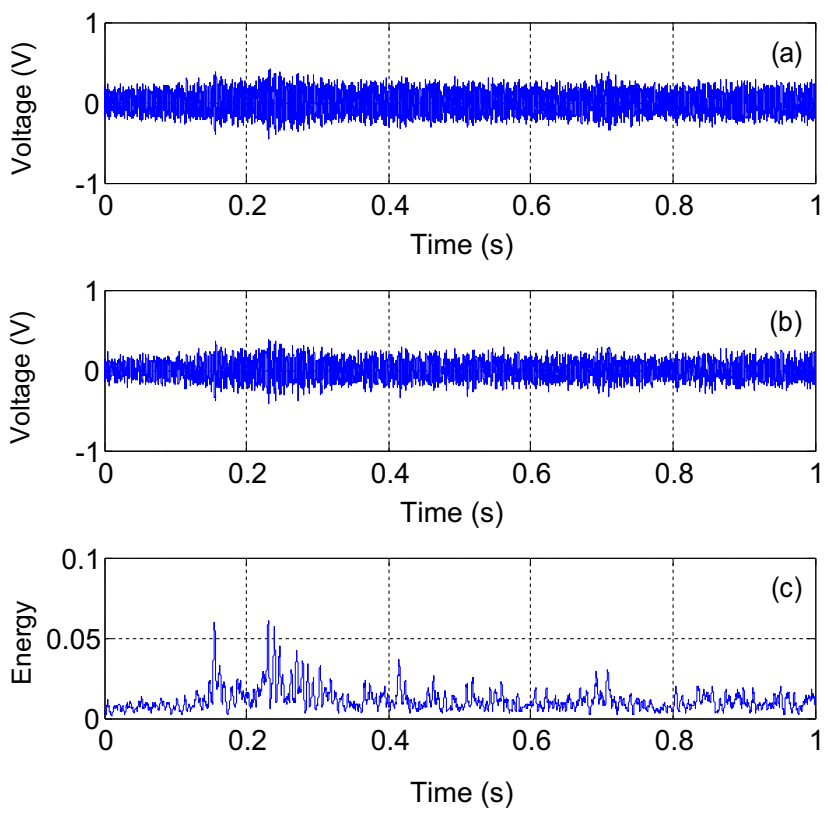

Fig. 8. (a) Original wave of signal 3, (b) Signal 3 after wavelet packet filtering, (c) Short time energy of signal 3. 

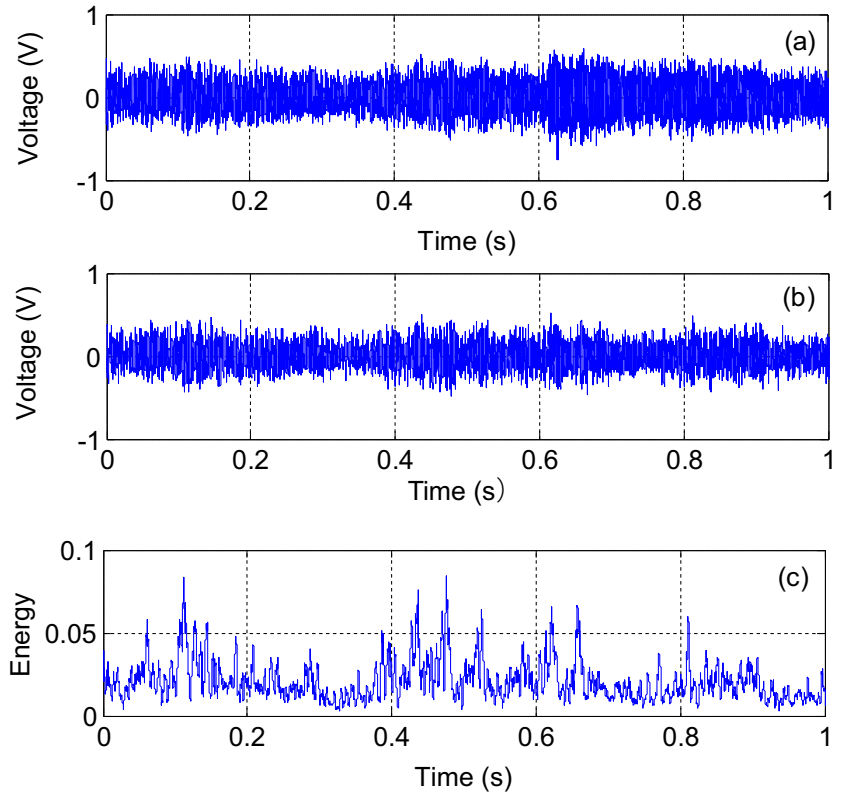

Fig. 9. (a) Original wave of signal 4, (b) Signal 4 after wavelet packet filtering, (c) Short time energy of signal 4.

(c) was stable without large energy variation. The amplitude of the energy variation was below 0.01 .

In Fig. 7 which showed the corresponding figures of signal 2, the energy had clear increasing at $0.3 \mathrm{~s}, 0.68 \mathrm{~s}$ and $0.9 \mathrm{~s}$. In addition, the amplitudes of the short time energy were around 0.2 . Hence, overall short time energy was not large and this signal was also a stable one.
Fig. 8 showed the corresponding figures of signal 3. It can be found that the stability of this signal was low. There were an unstable zone from $0.15 \mathrm{~s}$ to $0.3 \mathrm{~s}$ shown in Fig. 8(b). At the same moment the short time energy shown in Fig. 8(c) had a large variation with larger than 0.5 of amplitude.

The corresponding figures of signal 4 was shown in Fig. 9. The stability of this signal was worse than that of others. The unstable phenomena occurred at $0.1 \mathrm{~s}$ and a zone from $0.42 \mathrm{~s}$ to $0.55 \mathrm{~s}$. Under the unstable situation, the short time energy had larger variation with about 0.8 of amplitude.

Then corresponding analyses were conducted for above four signals. The possibilities and CDF were calculated and the results were shown in Fig. 10. It can be found that in Fig. 10(a), which showed the result of signal 1 , the majority of energies were below 0.01 , and the maximum possibility occurred at 0.001 of energy, which corresponded about 0.46 of possibility. According to the CDF curve, it had a large slope and approached a straight line. At 0.01 of the energy, the cumulative possibility approached $100 \%$. It showed that the short time energy were much focused and had a very small variation range.

Fig. 10(b) showed the result of signal 2. We can see that the range of signal distribution was a bit wider than that of signal 1 . The energy was in the zone from 0 to 0.02 . The maximum possibility appeared at 0.004 of energy with corresponding 0.18 of possibility. In addition, when the short time energy reached about 0.02 , the cumulative possibility achieved $100 \%$. The slope of CDF curve was also steep, which meant the energy distribution was focused. Even though there were large energies appearing, the overall energy variation range was still very limited.

In Fig. 10(c) which showed the result of signal 3, the energy distribution range was in the zone from 0 and 0.03 , which was much wider than that of signal 1 and 2 . The maximum possibility was about 0.1 and appeared when the energy was 0.009 . It meant that the energy distribution was further unfocused. When the energy
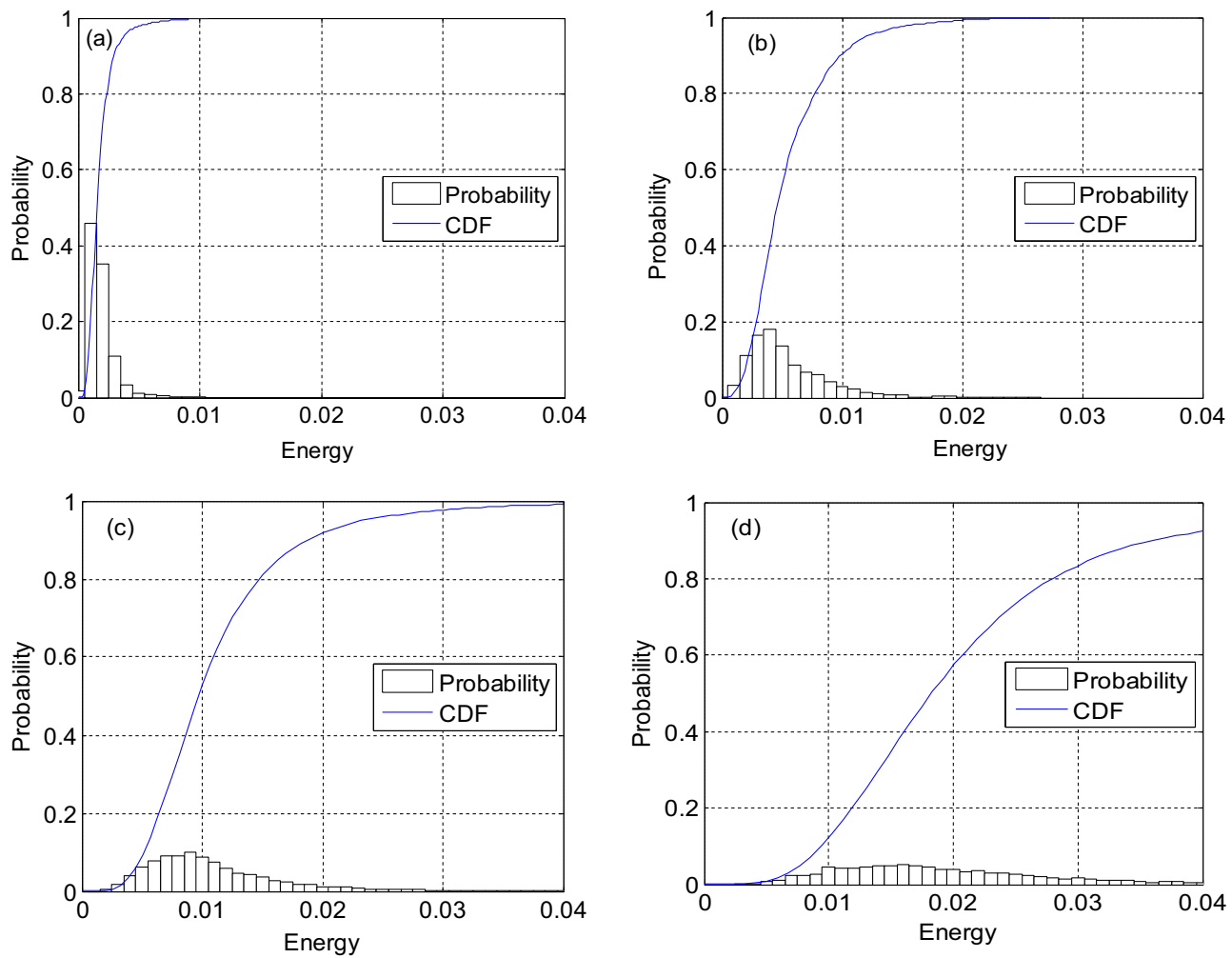

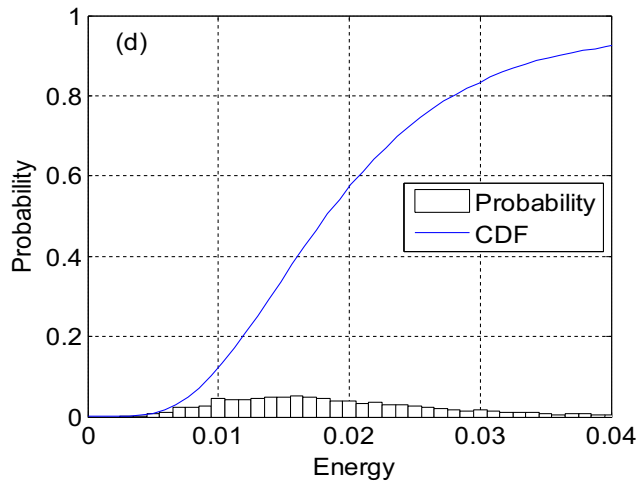

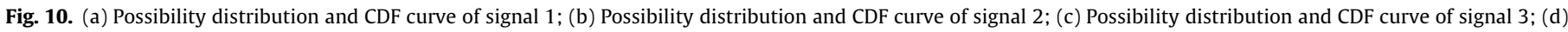
Possibility distribution and CDF curve of signal 4. 
Table 3

Evaluation results of the four signals.

\begin{tabular}{llll}
\hline Item & $E_{n C D F}$ & $E_{n P}$ & $A S S T$ \\
\hline 1 & 449.9796 & 0.3526 & 158.6693 \\
2 & 119.7916 & 0.1809 & 21.6658 \\
3 & 70.1784 & 0.0792 & 5.5611 \\
4 & 36.9689 & 0.0110 & 0.4083 \\
\hline
\end{tabular}

was about 0.04 , the cumulative possibility achieved at $99 \%$. The corresponding CDF curve ascended with a relative smooth slope.

The result of signal 4 was showed in Fig. 10(d). The energy distribution was much more unfocused with larger range. The maximum possibility, whose value was 0.02 , appeared when the energy was 0.016 . In the zone of the energy from 0.01 to 0.02 , the energy distribution was relative uniform, and majority were between 0.4 and 0.5 . According to the CDF curve, the cumulative possibility achieved at $92.3 \%$ when the energy was about 0.04 , which meant that about $7.7 \%$ short time energy were above 0.04 . The CDF curve ascended gently with a large curvature.

According to Fig. 10 and corresponding analyses, the stabilities of the four signals were worse by worse from signal 1 to signal 4 . However, the method cannot quantitatively describe the level of stability of welding process, to achieve this goal and clearly serve the actual application, the criterion mentioned in Section 3 was also employed for these four signals.

The self-designed statistical analysis criterion were employed to evaluate the stability of the four signals, as shown in Table 3. It can be seen that the values of ASSTs were worse by worse, which reasonably represented the stabilities of these four signals and validated the proposed method in this work.

\subsection{Extensive signal evaluation}

Many other experiments with different welding conditions were conducted to sufficiently validate the proposed methods. We chose 20 stable signals, 20 ordinary signals and 20 unstable signals to make further analysis. To achieve the hopeful effects, different welding conditions were employed. Then the corresponding results were included in Table 4.

Corresponding scatter figure of the ASST values of 60 samples in Table 4 was shown in Fig. 11. According to this figure and Table 2. It can be observed that the unstable signals had smaller values of ASST, the majority of values were below 10 . The values of ASST of

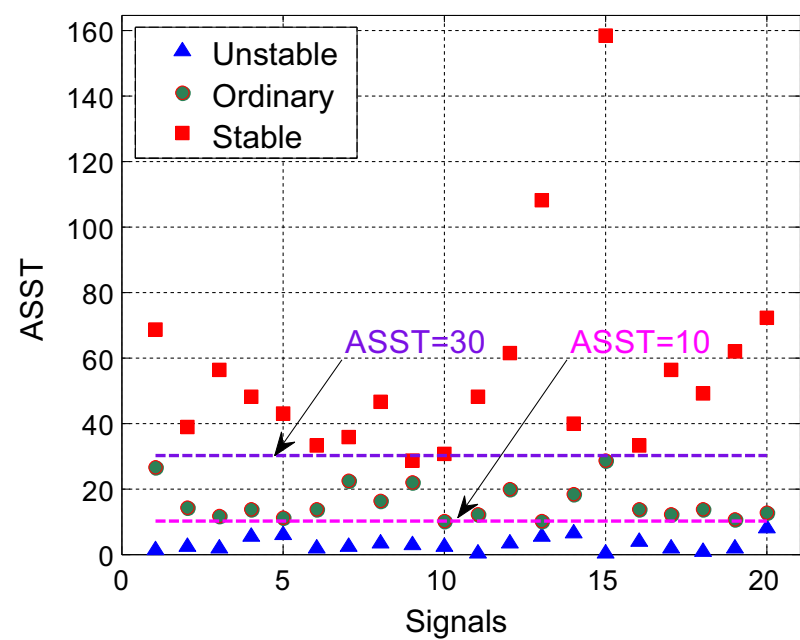

Fig. 11. Scatter figure of 60 signals.

ordinary signals were between 10 and 30 , while the values of stable signals were above 30 . As the signals more and more stable, the values of ASST were increasing.

\section{Conclusion and future work}

Short time energy can be employed to qualitatively monitor the stability of the arc sound signal, then to online analyze the stability of the arc welding process. Following conclusions can be drown:

1. The proposed method is based on the short time energy of the collected arc sound signals, because the signals include many useful information, it can be employed to detect the physical characteristics or process features during the arc welding process.

2. The CDF curve can effectively describe the stability of the arc sound signal. For the stable signal, the energy distribution is so focused, and the possibility of the max energy appearing is very small. On the other hand, the unstable signal presents an opposite tendency.

3. The significant distinction of the curve can be employed to monitor the stability of the signal, then extend to monitor the stability of the welding process. For providing an easyemployed result, a self-designed quantitative criterion is

Table 4

ASST results of 60 signals.

\begin{tabular}{|c|c|c|c|c|c|}
\hline Item & ASST for Unstable Signal & Item & ASST for Ordinary Signal & Item & ASST for Stable Signal \\
\hline 1 & 1.3364 & 1 & 26.6443 & 1 & 68.4521 \\
\hline 2 & 2.1908 & 2 & 14.3075 & 2 & 38.8904 \\
\hline 3 & 1.98754 & 3 & 11.742 & 3 & 56.3873 \\
\hline 4 & 5.2705 & 4 & 13.637 & 4 & 48.2751 \\
\hline 5 & 5.7213 & 5 & 10.9353 & 5 & 42.8994 \\
\hline 6 & 1.8123 & 6 & 13.7674 & 6 & 33.224 \\
\hline 7 & 2.2705 & 7 & 22.3382 & 7 & 35.637 \\
\hline 8 & 3.3809 & 8 & 15.9924 & 8 & 46.4751 \\
\hline 9 & 2.8879 & 9 & 21.6658 & 9 & 28.4391 \\
\hline 10 & 2.1499 & 10 & 9.8979 & 10 & 30.4348 \\
\hline 11 & 0.3336 & 11 & 11.9426 & 11 & 48.1409 \\
\hline 12 & 3.3809 & 12 & 19.5921 & 12 & 61.5255 \\
\hline 13 & 5.5611 & 13 & 9.8915 & 13 & 108.0445 \\
\hline 14 & 6.1822 & 14 & 18.4016 & 14 & 39.5841 \\
\hline 15 & 0.0776 & 15 & 28.3971 & 15 & 158.6693 \\
\hline 16 & 4.0354 & 16 & 13.8583 & 16 & 32.9248 \\
\hline 17 & 1.7028 & 17 & 12.2041 & 17 & 56.2789 \\
\hline 18 & 0.9627 & 18 & 13.8159 & 18 & 49.0976 \\
\hline 19 & 1.9578 & 19 & 10.4518 & 19 & 62.0679 \\
\hline 20 & 7.8567 & 20 & 12.813 & 20 & 72.3249 \\
\hline
\end{tabular}


proposed. Sufficient experimental results showed that the reliability was much higher and this method can be used to serve the quantitatively evaluate the stability of arc welding process.

4. Compared to previous contributions, based on their conclusions that the arc sound signal contains information which can reflect the variation characteristics of the welding process, the proposed method successfully realized quantitatively estimating the stability of arc welding process. The contribution in this work can benefit to the current arc welding academic research and actual production.

In future work, the evaluation method will be further improved, and we will explore using different criterions to evaluate the stability of arc sound signal, in addition, the effect of different operation parameters on the evaluation results will also be seriously considered. We hope this new method can benefit the academic research and actual arc welding process.

\section{Acknowledgements}

The authors would like to thank the Foundation for Public Welfare Research and Capacity Building Project of Guangdong Province (2015A010104010), Natural Science Foundation of Guangdong Province (2015A030313663) and China Postdoctoral Science Foundation (2016M602461).

\section{References}

[1] K. He, Q. Li, J. Chen, An arc stability evaluation approach for SW AC SAW based on Lyapunov exponent of welding current, Measurement 46 (1) (2013) 272 278.

[2] M. Dinham, G. Fang, Autonomous weld seam identification and localisation using eye-in-hand stereo vision for robotic arc welding, Robot. Comput.-Integr. Manuf. 29 (5) (2013) 288-301.

[3] M. Ron, P. Burget, O. Fiala, Identification of operations at robotic welding lines, in: 2015 IEEE International Conference on Automation Science and Engineering (CASE), 2015, pp. 470-476.

[4] J.F. Wang, B. Chen, H.B. Chen, S.B. Chen, Analysis of arc sound characteristics for gas tungsten argon welding, Sensor Rev. 29 (3) (2009) 240-249.

[5] N. Lv, Y. Xu, Z. Zhang. J. Wang, B. Chen, S. Chen, Audio sensing and modeling of arc dynamic characteristic during pulsed Al alloy GTAW process, Sensor Rev. 33 (2) (2013) 141-156.

[6] N. Lv, Y.L. Xu, G. Fang, H. Zhao, S.B. Chen, Mechanism Analysis and Feature Extraction of Arc Sound Channel for Pulse GTAW Welding Dynamic Process, Robotic Welding, Intelligence and Automation, Springer International Publishing, 2015.
[7] K. Pal, S. Bhattacharya, S.K. Pal, Investigation on arc sound and metal transfer modes for on-line monitoring in pulsed gas metal arc welding, J. Mater. Process. Technol. 210 (10) (2010) 1397-1410.

[8] L. Grad, J. Grum, I. Polajnar, Feasibility study of acoustic signals for on-line monitoring in short circuit gas metal arc welding, Int. J. Mach. Tools Manuf 44 (5) (2004) 555-561.

[9] L. Liu, T. Shimamura, Pitch-synchronous linear prediction analysis of highpitched speech using weighted short-time energy function, J. Signal Process. 19 (2) (2015) 55-66.

[10] N. Erdol, C. Castelluccia, A. Zilouchian, Recovery of missing speech packets using the short-time energy and zero-crossing measurements, IEEE Transact. Speech Audio Process. 1 (3) (1993) 295-303.

[11] M. Jalil, F.A. Butt, A. Malik, Short-time energy, magnitude, zero crossing rate and autocorrelation measurement for discriminating voiced and unvoiced segments of speech signals, in: Technological Advances in Electrical, Electronics and Computer Engineering (TAEECE), 2013 International Conference on, 2013, pp. 208-212.

[12] N. Raju, S. Mathini, T.L. priya, P. Preethi, M. Chandrasekar, Identifying the population of animals through pitch,formant, short time energy-A sound analysis, in: 2012 International Conference on Computing, Electronics and Electrical Technologies(ICCEET), Kumaracoil, 2012, pp. 704-709.

[13] S.K. Banchhor, A. Khan, Musical instrument recognition using zero crossing rate and short-time energy, Int. J. Appl. Inform. Syst. 1 (3) (2012) 16-19.

[14] S. Poornima, Basic characteristics of speech signal analysis, Int. J. Innovat. Res. Develop. 5 (4) (2016) 169-173.

[15] M.-H. Chun, S.-J. Han, N.-I. Tak, An uncertainty importance measure using a distance metric for the change in a cumulative distribution function, Reliab. Eng. Syst. Safety 70 (3) (2000) 313-321.

[16] S.A. Durai, E.A. Saro, Image compression with back-propagation neural network using cumulative distribution function, World Acad. Sci., Eng. Technol. 17 (2006) 60-64.

[17] D. Park, H. Seo, H. Kwon, B.G. Lee, Wireless packet scheduling based on the cumulative distribution function of user transmission rates, IEEE Transact. Commun. 53 (11) (2005) 1919-1929.

[18] E. Scheirer, M. Slaney, Construction and evaluation of a robust multifeature speech/music discriminator, in: Acoustics, Speech, and Signal Processing, 1997. ICASSP-97, 1997 IEEE International Conference on Munich, 1997, pp. $1331-1334$.

[19] D. Panton, Cumulative distribution function values for symmetric standardized stable distributions, Commun. Stat. - Simul. Comput. 21 (2) (1992) 485-492.

[20] P. Yao, K. Zhou, Q. Zhu, Quantitative evaluation method of arc sound spectrum based on sample entropy, Mech. Syst. Signal Process. 92 (2017) 379-390.

[21] P. Yao, J. Xue, K. Zhou, Study on the wirefeed speed prediction of double-wirepulsed MIG welding based on support vector machine regression, Int. J. Adv. Manuf. Technol. 79 (2015) 2107-2116.

[22] Hot-rolled plates and strips of carbon structural steels and high strength low alloy structural steels. China: Standardization Administration of the People's Republic of China; GB/T 3274-1988, 2008.

[23] Steel wires for melt welding. China: Standardization Administration of the People's Republic of China; GB/T 14957-94, 1995.

[24] J. Han, W. Ren, Z. Sun, Wavelet packet based damage identification of beam structures, Int. J. Solids Struct. 42 (26) (2005) 6610-6627. 